\title{
Bayesian Variable Selection for Finite Mixture Model of Linear Regressions
}

\author{
Kuo-Jung Lee ${ }^{\mathrm{a}}$, Ray-Bing Chen ${ }^{\mathrm{a}, *}$, Ying Nian $\mathrm{Wu}^{\mathrm{b}}$ \\ ${ }^{a}$ Department of Statistics, National Cheng Kung University \\ ${ }^{b}$ Department of Statistics, University of California, Los Angeles
}

\begin{abstract}
We propose a Bayesian variable selection method for fitting the finite mixture model of linear regressions. The model assumes that the observations come from a heterogeneous population which is a mixture of a finite number of sub-populations. Within each sub-population, the response variable can be explained by a linear regression on the predictor variables. If the number of predictor variables is large, it is assumed that only a small subset of variables are important for explaining the response variable. It is further assumed that for different sub-populations, different subsets of variables may be needed to explain the response variable. This gives rise to a complex variable selection problem. We propose to solve this problem within the Bayesian framework where we introduce two sets of latent variables. The first set of latent variables are membership indicators of the observations, indicating which sub-population each observation comes from. The second set of latent variables are inclusion/exclusion indicators for the predictor variables, indicating whether or not a variable is included in the regression model of a sub-population. Variable selection can then be accomplished by sampling from the posterior distributions of the indicators as well as the coefficients of the selected variables. We conduct simulation studies to demonstrate that the proposed method performs well in comparison with existing methods. We also analyze a real data set to further illustrate the usefulness of the proposed method.
\end{abstract}

Keywords: Gibbs sampler, Median probability criterion, Sparsity, Stochastic search variable selection

\section{Introduction}

Variable selection is a fundamental problem in linear regression. This problem becomes increasingly important in modern applications where a large number of variables are collected and it is often the case that the number of variables exceeds the number of observations. During the past decade, a rich literature has been developed around this problem. The methods proposed for this problem can be roughly classified into two categories.

One category consists of various penalized least squares methods, including the famous Lasso method (Tibashirani, 1996) based on the convex $\ell_{1}$ penalty for regularization, as well as non-convex

\footnotetext{
${ }^{*}$ Corresponding author

Email addresses: kjlee@stat.ncku.edu.tw (Kuo-Jung Lee), rbchen@stat.ncku.edu.tw (Ray-Bing Chen ), ywu@stat.ucla.edu (Ying Nian Wu)
} 
penalties such as SCAD (Fan and Li , 2001) and MCP (Zhang, 2010). The Lasso approach has also been extended to more sophisticated forms such as the group Lasso and graphical Lasso, see (Tibshirani, 2011) for a review.

The other category consists of various Bayesian variable selection methods, such as stochastic search variable selection (SSVS) (George and McCulloch, 1993, Chen, 2012) and Bayesian Lasso (Park and Casella , 2008). The Bayesian method assumes a prior distribution on the regression coefficients. A popular prior is the so-called "spike and slab" prior, which is a mixture of a point mass at zero and a diffused Gaussian distribution. Such a prior distribution assumes a binary indicator for each variable, indicating whether a variable is included in the regression or not. Variable selection can be accomplished by sampling from the posterior distributions of the latent indicators as well as the coefficients of the selected variables.

The two categories of methods are related in the sense that the penalty terms correspond to specific Bayesian prior distributions. The penalized least squares approaches, especially the Lasso and its extensions, usually enjoy the computational advantage in that the objective functions are convex and can be easily minimized.

Despite the wide applicability of the linear regression model powered by the modern variable selection tools, a single regression model can be inadequate if the data come from a heterogeneous population that consists of a number of different sub-populations with different characteristics. In this situation, it is possible that a separate linear regression model is needed for each sub-population. Moreover, the regression models in different sub-populations may use different subsets of predictor variables (or regressors, covariates) to explain the response variable. If the memberships of the observations are unobserved, then we naturally have a finite mixture model of linear regressions, where each mixture component is a linear regression model with its own subset of predictor variables. This gives rise to a variable selection problem that is more complex than that of a single linear regression model.

To solve the variable selection problem in the mixture model, one may extend the penalized least squares methods to the penalized likelihood of mixture models (Khalili and Chen, 2007; Städler, et al. 2010). However, the negative log-likelihood of the mixture model is no longer convex, thus losing one of the most appealing features of the Lasso method and its various extensions. As a result, one can only draw inference based on local minima of the objective functions. This difficulty motivates us to adopt the Bayesian alternative, which appears more natural and the Markov chain Monte Carlo (MCMC) algorithm may have a chance to escape the traps of local minima.

Specifically, the Bayesian variable selection method for the mixture model involves two sets of latent variables or indicators. The first set of latent variables are membership indicators associated with the observations, indicating which sub-population each observation comes from. The second set of latent variables are inclusion/exclusion indicators for the variables, where, for each subpopulation or mixture component, a binary indicator is associated with each predicator variable, indicating whether or not this variable is included in the linear regression of this mixture component. Variable selection, clustering, and parameter estimation can then be carried out by sampling from the posterior distributions of the indicators as well as the coefficients of the selected variables. Our simulation studies show that the Bayesian method performs well in comparison with existing methods. We also analyze a real data set to further illustrate the usefulness of the proposed method.

The rest of the article is organized as follows. Section 2 presents the finite mixture model of linear regressions and its Bayesian treatment, including the prior specifications and the MCMC algorithm for posterior sampling. Section 3 illustrates our method by simulation studies, where our method is compared with existing methods. Section 4 describes our analysis of a real data set 
to further illustrate our method. Section 5 discusses implementation issues concerning posterior inference, MCMC sampling and model selection criteria. Finally Section 6 concludes with a brief discussion.

\section{Finite mixture model of linear regressions}

Let $\left(y_{i}, x_{i}\right), i=1, \ldots, n$, be a data set of $n$ observations that come from a heterogeneous population, where $y_{i}$ is the response variable of the $i$-th observation, and $x_{i}=\left(x_{i 1}, \ldots, x_{i p}\right)^{\prime}$ collects the $p$ predictor variables or covariates of the $i$-th observation. We assume that the heterogeneous population consists of $M$ sub-populations or mixture components, and within each sub-population, $\left(y_{i}, x_{i}\right)$ follows a separate linear regression model. Specifically,

$$
y_{i} \mid\left(\rho_{m}, \beta_{m}, \sigma_{m}^{2}, m=1, \ldots, M\right) \sim \sum_{m=1}^{M} \rho_{m} \cdot N\left(x_{i}^{\prime} \beta_{m}, \sigma_{m}^{2}\right),
$$

where $\rho=\left(\rho_{1}, \ldots, \rho_{M}\right)$ is the proportion vector of the $M$ sub-populations, with $\rho_{m} \geq 0$ and $\sum_{m=1}^{M} \rho_{m}=1 . \beta_{m}=\left(\beta_{m 1}, \ldots, \beta_{m p}\right)^{\prime}$ is the coefficient vector for the linear regression in the $m$-th sub-population. $\sigma_{m}^{2}$ is the corresponding variance of the Gaussian residual errors.

\subsection{Two sets of latent variables}

As is standard for the mixture model, we introduce a latent variable $z_{i}$ for each observation $i$, so that $z_{i}=m$ indicates that the $i$-th observation comes from the $m$-th sub-population. Thus $P\left(z_{i}=m\right)=\rho_{m}$, i.e.,

$$
z_{i} \sim \operatorname{Multinomial}\left(\rho_{1}, \ldots, \rho_{M}\right), \text { and }\left[y_{i} \mid z_{i}=m\right] \sim N\left(x_{i}^{\prime} \beta_{m}, \sigma_{m}^{2}\right)
$$

In modern applications of linear regression models, the number of predictors $p$ can be large, and it is often assumed that the coefficient vector is sparse, i.e., only a small number of its components are different from zero. In other words, only a small number of predictor variables are to be included in the regression model. We assume that this is the case with the linear regression models of the sub-populations. We further assume that the sparsity patterns of the coefficient vectors of different sub-populations can be different from each other, i.e., in the linear regression models of different sub-populations, different subsets of variables may be selected to explain the response variable. The goal of inference is to infer the sparse coefficient vectors of the sub-populations while classifying the observations into the sub-populations.

To facilitate variable selection, we introduce a latent vector $r_{m}=\left(r_{m 1}, \ldots, r_{m p}\right)^{\prime}$ for each subpopulation $m . r_{m j}$ is an inclusion/exclusion indicator, so that $\beta_{m j}=0$ if $r_{m j}=0$ and $\beta_{m j} \neq 0$ if $r_{m j}=1$. Let $\beta_{m}\left(r_{m}\right)$ collect all the non-zero elements of $\beta_{m}$ and let $x_{i}\left(r_{m}\right)$ be the active elements of $x_{i}$ corresponding to those elements of $r_{m}$ that are equal to 1 . The model in equation (1) can then be expressed as

$$
y_{i} \mid\left(\rho_{m}, \beta_{m}, \sigma_{m}^{2}, r_{m}, m=1, \ldots, M\right) \sim \sum_{m=1}^{M} \rho_{m} \cdot N\left(x_{i}^{\prime}\left(r_{m}\right) \beta_{m}\left(r_{m}\right), \sigma_{m}^{2}\right)
$$


With the two sets of latent variables $\left\{z_{i}, i=1, \ldots, n\right\}$ and $\left\{r_{m}=\left(r_{m j}, \ldots, r_{m p}\right), m=1, \ldots, M\right\}$, the Bayesian computation can be conveniently conducted. In the following subsections, we shall introduce the prior assumptions first and then present the details of the Bayesian analysis.

\subsection{Prior distributions}

First consider the probability vector $\rho$ of mixture proportions. Similar to Viele and Tong (2002), we assumes a conjugate Dirichlet prior distribution on $\rho$,

$$
\rho \sim \operatorname{Dirichlet}\left(\alpha_{1}, \ldots, \alpha_{M}\right) .
$$

In each mixture component of the regression model, the prior distributions of the indicator variables $r_{m j}$ are assumed to be independent Bernoulli $\left(d_{m j}\right)$ for $j=1, \ldots, p$. As a result, the joint distribution of $r_{m}=\left(r_{m 1}, \ldots, r_{m p}\right)^{\prime}$ is

$$
\pi\left(r_{m}\right)=\prod_{j=1}^{p} d_{m j}^{r_{m j}}\left(1-d_{m j}\right)^{1-r_{m j}} .
$$

Now consider the prior distribution of the coefficient vector $\beta_{m}$. Let $Y_{m}$ be the response vector consisting of responses in the $m$-th sub-population, and let $X_{m}\left(r_{m}\right)$ be the corresponding design matrix with rows consisting of $x_{i}^{\prime}\left(r_{m}\right)$ where the observation $i$ belongs to the sub-population $m$. Following Zellner (1996), the prior of $\beta_{m}\left(r_{m}\right)$ is assumed to be the $g$-prior which is given by

$$
\beta_{m}\left(r_{m}\right) \sim N\left(\hat{\beta}_{m}\left(r_{m}\right), g_{m} \sigma_{m}^{2}\left[X_{m}^{\prime}\left(r_{m}\right) X_{m}\left(r_{m}\right)\right]^{-1}\right),
$$

where $\hat{\beta}_{m}\left(r_{m}\right)=\left[X_{m}^{\prime}\left(r_{m}\right) X_{m}\left(r_{m}\right)\right]^{-1} X_{m}^{\prime}\left(r_{m}\right) Y_{m}$ and $g_{m}$ is a positive number. To avoid the problem of a singular $X_{m}^{\prime}\left(r_{m}\right) X_{m}\left(r_{m}\right)$, we can replace $\left[X_{m}^{\prime}\left(r_{m}\right) X_{m}\left(r_{m}\right)\right]^{-1}$ by $\left[X_{m}^{\prime}\left(r_{m}\right) X_{m}\left(r_{m}\right)+\lambda_{m} I\right]^{-1}$, where $\lambda_{m}$ is a positive number called the ridge parameter (Gupta and Ibrahim, 2007, Baragatti and Pommeret, 2012), and $I$ is the identity matrix. Thus we have a modified $g$-prior distribution given as follows

$$
\beta_{m}\left(r_{m}\right) \sim N\left(\hat{\beta}_{m}^{\lambda_{m}}\left(r_{m}\right), g_{m} \sigma_{m}^{2}\left[X_{m}^{\prime}\left(r_{m}\right) X_{m}\left(r_{m}\right)+\lambda_{m} I\right]^{-1}\right),
$$

where $\hat{\beta}_{m}^{\lambda_{m}}\left(r_{m}\right)=\left(X_{m}^{\prime}\left(r_{m}\right) X_{m}\left(r_{m}\right)+\lambda_{m} I\right)^{-1} X_{m}^{\prime}\left(r_{m}\right) Y_{m}=w_{m}\left(r_{m}\right) X_{m}^{\prime}\left(r_{m}\right) Y_{m}$ with $w_{m}\left(r_{m}\right)=$ $\left(X_{m}^{\prime}\left(r_{m}\right) X_{m}\left(r_{m}\right)+\lambda_{m} I\right)^{-1}$. Note that $\hat{\beta}_{m}\left(r_{m}\right)=\hat{\beta}_{m}^{\lambda_{m}}\left(r_{m}\right)$ if we set $\lambda_{m}=0$. Finally, for the prior of each $\sigma_{m}^{2}$, we assume $\sigma_{m}^{2} \sim \operatorname{IG}\left(\frac{a_{m_{0}}}{2}, \frac{b_{m_{0}}}{2}\right)$ independently.

We assume that $z_{i}$ 's are independent of $\left(\gamma_{m}, \beta_{m}, \sigma_{m}\right)$, and $\left(\gamma_{m}, \beta_{m}, \sigma_{m}\right)$ are assumed to be independent of each other. For each $m, \gamma_{m j}$ 's are assumed to be independent of each other.

\subsection{Gibbs sampler scheme for variable selection}

With the prior specification described in the above subsection, the joint posterior distribution is derived as follows. The complete-data likelihood of the mixture regression model for $y=\left(y_{1}, \ldots, y_{n}\right)$ and $z=\left(z_{1}, \ldots, z_{n}\right)$ is

$$
\ell(y, z \mid \theta)=\prod_{i=1}^{n} \rho_{z_{i}} f\left(y_{i} \mid \theta_{z_{i}}\right)=\prod_{m=1}^{M} \rho_{m}^{n_{m}}\left[\prod_{i \in G_{m}} f\left(y_{i} \mid \theta_{z_{i}}\right)\right],
$$


where $n_{m}=\sum_{i=1}^{n} I\left\{z_{i}=m\right\} . G_{m}$ is the set containing the members in the mixture component $m$. $\theta_{m}=\left(\beta_{m}, \sigma_{m}^{2}, \rho_{m}, r_{m}\right) . f\left(y_{i} \mid \theta_{m}\right)$ is the probability density function of a Gaussian random variable with mean $x_{i}^{\prime}\left(r_{m}\right) \beta_{m}\left(r_{m}\right)$ and variance $\sigma_{m}^{2}$. Let $\theta=\left(\theta_{1}, \ldots, \theta_{m}\right)$. Combining the complete-data likelihood and the prior $\pi(\theta)$, we have the posterior distribution given by

$$
p(\theta, z \mid y) \propto \prod_{m=1}^{M} \rho_{m}^{n_{m}}\left[\prod_{i \in G_{m}}\left[f\left(y_{i} \mid \theta_{z_{i}}\right)\right]\right] \pi(\theta) .
$$

We use the Gibbs sampler to generate the parameters from the posterior distribution. To implement the Gibbs sampler, we need to derive the full conditional distributions of all the components of $\theta$ and $z$ separately. Starting from an initial value, the Gibbs sampler proceeds by iteratively drawing samples from the following conditional distributions:

Step 1 The conditional distribution of $z$ given $\theta$ and $y$ is, for $i=1, \ldots, n$,

$$
P\left(z_{i}=m \mid \theta, y\right)=\frac{\rho_{m} f\left(y_{i} \mid \theta_{m}\right)}{\sum_{m=1}^{M} \rho_{m} f\left(y_{i} \mid \theta_{m}\right)} .
$$

Step 2 The conditional distribution of $\rho$ given $z$ is

$$
\rho \sim \operatorname{Dirichlet}\left(n_{1}+\alpha_{1}, \ldots, n_{M}+\alpha_{M}\right) .
$$

Step 3 The conditional distribution of $\sigma_{m}^{2}$ given $\beta_{m}, r_{m}, z, y$ is $\sigma_{m}^{2} \sim I G\left(\frac{a_{m}}{2}, \frac{b_{m}}{2}\right)$, where $q_{m}=$ $\sum_{j=1}^{p} r_{m j} ; a_{m}=n_{m}+q_{m}+a_{m_{0}}$, and

$$
\begin{aligned}
b_{m}= & {\left[Y_{m}-X_{m}^{\prime}\left(r_{m}\right) \beta_{m}\left(r_{m}\right)\right]^{\prime}\left[Y_{m}-X_{m}^{\prime}\left(r_{m}\right) \beta_{m}\left(r_{m}\right)\right] } \\
& +\frac{\left[\beta_{m}\left(r_{m}\right)-\hat{\beta}_{m}^{\lambda_{m}}\left(r_{m}\right)\right]^{\prime} w_{m}^{-1}\left(r_{m}\right)\left[\beta_{m}\left(r_{m}\right)-\hat{\beta}_{m}^{\lambda_{m}}\left(r_{m}\right)\right]}{n_{m}}+b_{m_{0}} .
\end{aligned}
$$

Step 4 The conditional distribution of $\beta_{m}$ given $\sigma_{m}^{2}, r_{m}, z, y$ is

$$
\beta_{m}\left(r_{m}\right) \sim N\left(\mu_{m}, \Omega_{m}\right),
$$

where $\mu_{m}=\Omega_{m}\left(n_{m} X_{m}\left(r_{m}\right) Y_{m}^{\prime}+w_{m}\left(r_{m}\right)^{-1} \hat{\beta}_{m}^{\lambda_{m}}\left(r_{m}\right)\right) / n_{m} \sigma_{m}^{2}$ and $\Omega_{m}^{-1}=\left[\frac{n_{m} X_{m}^{\prime}\left(r_{m}\right) X_{m}\left(r_{m}\right)+w_{m}^{-1}\left(r_{m}\right)}{n_{m} \sigma_{m}^{2}}\right]$.

Step 5 The conditional distribution of $r_{m}$ given $\beta_{m}, \sigma_{m}^{2}, z, y$ is, for each $j$ in $G_{m}$,

$$
p\left(r_{m j}=1 \mid r_{m(-j)}, z, y\right)=\frac{p\left(r_{m j}=1 \mid r_{m(-j)}, z, y\right)}{p\left(r_{m j}=1 \mid r_{m(-j)}, z, y\right)+p\left(r_{m j}=0 \mid r_{m(-j)}, z, y\right)},
$$

where $r_{m(-j)}$ is the vector obtained from $r_{m}$ by excluding $r_{m j}$.

\subsection{Variable selection criterion and component assignment}

After a sufficient number, say $K$, of samples of parameters are drawn from the posterior distribution by the Gibbs sampler, they are used for posterior inference. In order to determine the active 
variables of the linear regression model of each sub-population, we collect the posterior samples of $r_{m j}$ 's and adopt the median probability criterion (Barbieri and Berger, 2004) for variable selection. Specifically, we calculate the relative frequency of the variable $x_{j}$ within the sub-population $m$ by

$$
\hat{p}\left(r_{m j}=1 \mid y\right)=\frac{1}{K} \sum_{k=1}^{K} I\left\{r_{m j}^{(k)}=1\right\},
$$

where $r_{m j}^{(k)}$ is the $k$-th posterior sample generated by the Gibbs sampler. This gives us an estimate of the posterior variable inclusion probability as a measure of the relative importance of the $j$-th predictor variable within the $m$-th sub-population or mixture component. Specifically, based on the median probability criterion, we claim that the variable $x_{j}$ is active for the $m$-th sub-population if

$$
\hat{p}\left(r_{m j}=1 \mid y\right) \geq 1 / 2 .
$$

The posterior samples of $z_{i}$ 's can be used for membership assignment of each observation $i$. Specifically, an observation $y_{i}$ is assigned to the $m$-th sub-population if

$$
\hat{p}\left(z_{i}=m \mid y\right)=\max _{g} \hat{p}\left(z_{i}=g \mid y\right)=\max _{g} \frac{1}{K} \sum_{k=1}^{K} I\left\{z_{i}^{(k)}=g\right\},
$$

where $z_{i}^{(k)}$ is the membership of $i$-th observation in the $k$-th posterior sample generated by the Gibbs sampling. $I\{\}$ is the indicator function.

\subsection{Identifiability}

A common issue with a mixture model is the nonidentifiability of the component parameters. This is due to the so-called "label-switching" problem (Celeux, 1998) caused by the symmetry in the likelihood of the model parameters. For a review of the identifiability issue in Bayesian mixture modeling, the interested reader can consult Jasra, et al. (2005) and the references therein. For an $M$-component mixture, the parameter space has $M$ ! regions over which the likelihood is identical, that is, the component parameters are not marginally identifiable. Thus, if $\left(\theta_{1}, \ldots, \theta_{M}\right)$ is a local maximum of the likelihood function, so is $\left(\theta_{\omega_{1}}, \ldots, \theta_{\omega_{M}}\right)$ for every permutation $\omega$. This makes maximization and exploration of the posterior distribution difficult.

A commonly adopted solution to this problem is to remove the symmetry by imposing an identifiability constraint on the parameters. In this paper, we arrange the mixture components in the order of increasing variances $\sigma_{1}^{2}<\sigma_{2}^{2}<\ldots<\sigma_{M}^{2}$. For a more detailed discussion and some examples of nonidentifiability, please see Frühwirth-Schnatter (2006).

\subsection{Number of sub-populations}

In the finite mixture model, the number $M$ of the mixture components or sub-populations is usually unknown, and mis-specification of the number of mixture components can lead to wrong results on variable selection as well as the classification of the observations. Instead of pre-specifying a fixed number of mixture components, several information criteria can be adopted to determine the number of components.

Most of the existing approaches address the issue of model selection using criteria such as the Akaike Information Criterion (AIC), Bayesian Information Criterion (BIC), and Deviance Information Criterion (DIC) (Celeux et al. 2006). Let $M$ be the possible number of components in the 
mixture model. Let $d_{M}$ be the number of parameters. Let $\hat{\vartheta}_{M}$ be the estimates of the parameters. The AIC, BIC and DIC for the mixture model with $M$ components are defined as, respectively,

$$
\begin{aligned}
& \mathrm{AIC}_{M}=-2 \log L\left(y \mid \hat{\vartheta}_{M}\right)+2 d_{M} ; \\
& \mathrm{BIC}_{M}=-2 \log L\left(y \mid \hat{\vartheta}_{M}\right)+d_{M} \log (n) ; \\
& \operatorname{DIC}_{M}=-2 \log L\left(y \mid \hat{\vartheta}_{M}\right)+p_{D},
\end{aligned}
$$

where $p_{D}$ is the posterior mean deviance minus deviance evaluated at the posterior mean of the parameters, which is used to measure the complexity in terms of the effective number of parameters. Evidently, BIC assigns more penalty to the complexity of the selected model. However, neither BIC or AIC take into account the fact that in a clustering context a finite mixture model is fitted with the hope of finding a good partition of the data. To overcome this shortcoming, the integrated classification of likelihood (ICL) criterion was proposed by Biernacki et al. (2000) with the specific aim of selecting the number of components of a finite mixture model in model-based clustering. McLachlan and Peel (2000) showed that when the number of members within each group is large enough, ICL is approximately equal to

$$
\mathrm{ICL}-\mathrm{BIC}_{M}=\mathrm{BIC}_{M}+2 \mathrm{EN}\left(\hat{\vartheta}_{M}\right)
$$

where $\operatorname{EN}\left(\hat{\vartheta}_{M}\right)=-\sum_{i=1}^{n} \sum_{m=1}^{M} P\left(z_{i}=m \mid y_{i}, \hat{\vartheta}_{M}\right) \log P\left(z_{i}=m \mid y_{i}, \hat{\vartheta}_{M}\right)$. This so-called entropy term is to measure the degree of the inability of the fitted $M$-component mixture model to provide a good partition of the data. It is close to 0 if the resulting clusters are well separated and will have a large value if this is not the case. Therefore, the ICL and its asymptotic variant penalize not only the complexity but also the failure of classification in well-separated clusters of the fitted model. We can select the model by minimizing $\mathrm{AIC}_{M}, \mathrm{BIC}_{M}$, ICL-BIC ${ }_{M}$, or $\mathrm{DIC}_{M}$. The performances of these criteria will be illustrated in the next section.

\section{Simulation study}

In this section we conduct simulation studies to illustrate the performance of the proposed method for fitting the mixture model of linear regressions. We shall also demonstrate that our method can be easily applied to the problem of $p>n$. The settings of our simulation studies are based on those in Städler, et al. (2010) and Khalili and Chen (2007). In both papers, the penalized least squares methods are used for variable selection. Specifically, given the log-likelihood function of the model (1), the penalty terms are added to induce sparsity, i.e.,

$$
l_{n}(\theta)=\sum_{i=1}^{n} \log \left(\sum_{m=1}^{M} \rho_{m} \cdot \phi\left(y_{i} ; x_{i}^{\prime} \beta_{m}, \sigma_{m}^{2}\right)\right)-\sum_{m=1}^{M} \pi_{m}\left(\sum_{j=1}^{p} p_{n m}\left(\beta_{m j}\right)\right)
$$

where $\phi\left(y ; \mu, \sigma^{2}\right)$ is the Gaussian density function, and $p_{n m}\left(\beta_{m j}\right)$ is a penalty function that is nonnegative and nondecreasing in $\left|\beta_{m j}\right|$, the absolute value of $\beta_{m j}$. Both papers develop EM-type algorithms for variable selection. We shall show that our method exhibits better performance in variable selection compared to the penalized least squares methods. 


\subsection{Simulation 1}

We first conduct a simulation study according to the setting of Khalili and Chen (2007). For simplicity, we consider the mixture model of two linear regressions, i.e. $M=2$, given by

$$
y \sim \rho N\left(x^{\prime} \beta_{1}, 1\right)+(1-\rho) N\left(x^{\prime} \beta_{2}, 1\right) .
$$

The predictor variables $x$ in equation (4) are simulated from a 5-dimensional multivariate normal distribution with mean vector $\mathbf{0}$ and covariance matrix $\Sigma$. Let $\Sigma(i, j)$ denote the $(i, j)$ entry of $\Sigma$. We set $\Sigma(i, j)=\pi^{|i-j|}$ where $-1<\pi<1$. We consider two correlation structures with $\pi=0$ and $\pi=0.5$ respectively. The regression coefficients are set to be $\beta_{1}=(1,0,0,3,0)$ and $\beta_{2}=(-1,2,0,0,3)$ respectively, the same as in Khalili and Chen (2007). Three different choices of mixing proportions $\rho=0.1,0.3,0.5$ are considered.

For the prior parameters, we first set the prior inclusion probabilities of the variables $d_{m j}=$ 0.5 for all variables $j$ and sub-populations $m$, to reflect the fact that we do not have any prior information about which variables are active in different sub-populations. In the inverse gamma prior for $\sigma_{m}^{2}$, the nearly non-informative priors are used by setting $a_{m_{0}}=b_{m_{0}}=0.001$ for all $\mathrm{m}$. We set $\alpha_{i}=2$ in the Dirichlet prior for $i=1$ and 2. For the value of $g_{m}$ in the $g$-prior, we set $g_{m}=n_{m}$. Since there is no singularity problem, we set the ridge parameter $\lambda_{m}=0$.

We run the Gibbs sampler 1,000,000 sweeps and collect the posterior samples. As advocated by Flegal et al. (2008), it is not necessary to have a burn-in period, thus we keep all the samples as our posterior samples for Bayesian inference. The median probability criterion (2) is used to decide if a variable is active or not. In fact, with 100,000 iterations, the marginal posterior probabilities in our simulation are very stable over random restarts, implying convergence. We also studied the performance with the burn-in step. Since the results are similar to these without burn-in step, they are not shown here due to the space limit. We will discuss this issue in Section 5.

To examine the performance of our method and compare our results to those in the paper of Khalili and Chen (2007), we calculate the average estimates of the number of correct zeros in the coefficient vectors and the corresponding standard errors over 100 simulated data sets with $n=100$. The results are given in Table 1. In this table, we also show the results of MixLasso and MixSCAD reported in Khalili and Chen (2007). Overall our approach (labeled as "Bayes" in the table) is superior to the MixLASSO and MixSCAD methods in terms of the correct identification rates, especially for the case of $\rho=0.1$. In addition, the proposed approach also produces lower incorrect identification rates in most cases. This shows that the proposed Bayesian variable selection approach performs better on variable selection than the two methods in Khalili and Chen (2007). 
Table 1: Average numbers of correct and incorrect zero coefficients with $n=100$. The numbers within parentheses are the standard errors of the corresponding estimates.

\begin{tabular}{|c|c|c|c|c|c|c|c|c|}
\hline \multirow[b]{3}{*}{ Method } & \multicolumn{4}{|c|}{$\pi=0$} & \multicolumn{4}{|c|}{$\pi=0.5$} \\
\hline & \multicolumn{2}{|c|}{ Component 1} & \multicolumn{2}{|c|}{ Component 2} & \multicolumn{2}{|c|}{ Component 1} & \multicolumn{2}{|c|}{ Component 2} \\
\hline & Correct & Incorrect & Correct & Incorrect & Correct & Incorrect & Correct & Incorrect \\
\hline$\rho=0.5$ & & & & & & & & \\
\hline Bayes & $2.98_{(0.01)}$ & $0.016_{(0.01)}$ & $1.99_{(0.005)}$ & $0.011_{(0.001)}$ & $2.97_{(0.02)}$ & $0.021_{(0.01)}$ & $1.99_{(0.005)}$ & $0.022_{(0.002)}$ \\
\hline MixSCAD & 2.98 & 0.021 & 1.99 & 0.024 & 2.94 & 0.024 & 1.98 & 0.058 \\
\hline $\begin{array}{l}\text { MixLASSO } \\
\rho=0.3\end{array}$ & 2.93 & 0.026 & 1.98 & 0.025 & 2.52 & 0.027 & 1.77 & 0.078 \\
\hline Bayes & $2.95_{(0.02)}$ & $0.051_{(0.02)}$ & $1.98_{(0.006)}$ & $0.016_{(0.002)}$ & $2.90_{(0.05)}$ & $0.055_{(0.02)}$ & $1.96_{(0.012)}$ & $0.025_{(0.003)}$ \\
\hline MixSCAD & 2.81 & 0.057 & 1.92 & 0.044 & 2.84 & 0.089 & 1.96 & 0.024 \\
\hline $\begin{array}{l}\text { MixLASSO } \\
\rho=0.1\end{array}$ & 2.74 & 0.060 & 1.94 & 0.028 & 2.54 & 0.133 & 1.78 & 0.042 \\
\hline Bayes & $2.90_{(0.04)}$ & $0.107_{(0.04)}$ & $1.95_{(0.010)}$ & $0.022_{(0.005)}$ & $2.85_{(0.10)}$ & $0.122_{(0.04)}$ & $1.94_{(0.018)}$ & $0.055_{(0.006)}$ \\
\hline MixSCAD & 2.10 & 0.246 & 1.88 & 0.023 & 2.40 & 0.577 & 1.99 & 0.026 \\
\hline MixLASSO & 2.64 & 0.733 & 1.83 & 0.040 & 2.58 & 0.919 & 1.71 & 0.044 \\
\hline
\end{tabular}




\subsection{Simulation 2}

We conduct another simulation study to illustrate the performance of our method in different scenarios to be described below. There are four purposes in this simulation study. First, we examine the ability of our approach to select the active variables even when the design matrix is highly correlated. Second, we investigate the influence of different values of $g_{m}$ of the $g$-prior on the posterior estimation and identification of the active variables. Third, we demonstrate that the information criteria can be used to determine the number of sub-populations. Forth, we apply our method to the problem of large $p$ and small $n$ by increasing the number of inactive variables.

In this simulation study, we consider the same setting as the model M4 in Städler, et al. (2010). Suppose that there are $M=3$ sub-populations with a total of 150 observations and the corresponding $\beta$ 's are $\beta_{1}=(3,3,0,0,0,0), \beta_{2}=(0,0,-2,-2,0,0)$, and $\beta_{3}=(0,0,0,0,-3,2)$ in the three regression models respectively. We set $\sigma_{m}^{2}=0.5$ for all the sub-populations $m$ and the mixing proportions are assumed to be $\rho=(1 / 3,1 / 3,1 / 3)$.

In the Bayesian analysis, except for the prior parameters $\lambda_{m}$ and $g_{m}$ in the $g$-prior, the other prior parameters are the same as those in Simulation 1. We again iterate the Gibbs sampler $1,000,000$ sweeps to generate the posterior samples. The median probability criterion (2) is used to determine which variables are active. The classification of each observation is determined according to $(3)$.

To evaluate the performance of our method, we consider the following measures: the True Classification Rate (TCR), the True Positive Rate (TPR), the False Positive Rate (FPR), and the rate of the True Classification of Observations (TCO). They are defined as follows

$$
\begin{aligned}
\mathrm{TCR} & =\frac{\text { number of correctly selected variables }}{\text { number of variables }} ; \\
\mathrm{TPR} & =\frac{\text { number of correctly selected variables }}{\text { number of active variables }} ; \\
\mathrm{FPR} & =\frac{\text { number of falsely selected variables }}{\text { number of inactive variables }} ; \\
\mathrm{TCO} & =\frac{\text { number of correct classifications of observations }}{\text { number of observations }} .
\end{aligned}
$$

TCR is an overall evaluation of the accuracy in the identification of the active and inactive variables. TPR is the average rate of active variables identified correctly and is used to measure the power of the method. FPR is the average rate of inactive variables that are included in the regression and it can be considered as the type I error rate of the selection approach. TCO is the rate that the observations are classified into the correct sub-populations. In these four measures, TCR, TPR, and TCO have the property that the larger they are, the better, but for FPR, the smaller it is, the better.

We generate the variables from 5-dimensional multivariate normal distribution with different pairwise correlations $\Sigma(i, j)=\pi^{|i-j|}$, where $\pi=0.25,0.5,0.75$, and 0.995 . Since we want to investigate the effect of $\lambda_{m}$ on selecting important variables in the case of multicollinearity, we fix $g_{m}=n_{m}$ as suggested by Zellner (1996). We start with $\lambda_{m}=\frac{1}{p}$ as suggested by Baragatti and Pommeret (2012), and then try $\lambda_{m}=\frac{1}{2 p}$ and $\frac{2}{p}$. Based on 100 replications, the results are shown in Table 2. The performances under different $\lambda_{m}$ are similar, so we choose $\lambda_{m}=\frac{1}{p}$ in the remaining experiments. Note that with increasing degree of collinearity between the variables, the correct identification rate is decreasing. 
Table 2: The measures of TCR, TPR, FPR, and TCO for different values of $\lambda_{m}$ and $g_{m}$.

\begin{tabular}{|c|c|c|c|c|c|c|c|c|c|c|c|c|}
\hline \multirow[b]{3}{*}{$\lambda_{m}$} & \multicolumn{12}{|c|}{$\pi$} \\
\hline & \multicolumn{3}{|c|}{0.25} & \multicolumn{3}{|c|}{0.5} & \multicolumn{3}{|c|}{0.75} & \multicolumn{3}{|c|}{0.995} \\
\hline & $\frac{1}{2 p}$ & $\frac{1}{p}$ & $\frac{2}{p}$ & $\frac{1}{2 p}$ & $\frac{1}{p}$ & $\frac{2}{p}$ & $\frac{1}{2 p}$ & $\frac{1}{p}$ & $\frac{2}{p}$ & $\frac{1}{2 p}$ & $\frac{1}{p}$ & $\frac{2}{p}$ \\
\hline TCR & 0.99 & 0.99 & 0.99 & 0.99 & 0.99 & 0.98 & 0.98 & 0.97 & 0.97 & 0.83 & 0.84 & 0.86 \\
\hline TPR & 1 & 1 & 1 & 1 & 1 & 0.98 & 0.98 & 0.97 & 0.97 & 0.67 & 0.58 & 0.58 \\
\hline FPR & 0.008 & 0.010 & 0.011 & 0.009 & 0.010 & 0.010 & 0.011 & 0.012 & 0.012 & 0.12 & 0.13 & 0.14 \\
\hline TCO & 0.92 & 0.91 & 0.91 & 0.92 & 0.91 & 0.90 & 0.92 & 0.91 & 0.90 & 0.92 & 0.92 & 0.92 \\
\hline
\end{tabular}

To demonstrate that our approach can work for the problem of large $p$ and small $n$, we consider $p=65,85,105,125,155$. We set $\beta_{m j}$ at the same values as in the previous simulation except $\beta_{m j}=0$ for $j=7, \ldots, p$. For all $m$ and the additional covariates, $x_{i}$ 's are independently generated from multivariate normal distribution with zero mean vector and identity covariance matrix. According to the previous experiments, we fix $\lambda_{m}=1 / p$ for all $m$. In this simulation study, we find that the value $g_{m}$ has an effect on the variable selection results. This is a typical phenomenon when $g$-prior is used. In general, smaller values of $g_{m}$ tend to result in more complex model, whereas larger values of $g_{m}$ tend to produce more parsimonious model. In our simulation study, we find that when $g_{m}=n_{m}$, some inactive variables are selected, especially in the large $p$ examples, for instance, $p>100$. So if the ratio of the number of variables to that of observations, $p / n$, is greater than or equal to 3 , we recommend setting $g_{m}=100 \times p \times M / n$. For each $p$, we repeat the simulation 100 times. The box plots of TPR, FPR and TCO for different numbers of covariates, $p$, are shown in Figure 1 . These box plots show that our method is quite good at identifying the true active variables and classifying the observations into the sub-populations. Moreover, the distribution of TCO indicates that most of the time the observations are correctly classified. As to the computational cost of the proposed approach, we record the average computing time for 10,000 iterations of MCMC with $\pi=0.5$ and different numbers of variables $p$ in this simulation study. The computation time is reported in Table 3. The computation is done on a Linux cluster containing Xeon E5620 2.4GHz 2 Quad-core, $16 \mathrm{~GB} \times 8=128 \mathrm{~GB}$.

Next, we evaluate the performances of AIC, BIC, ICL-BIC, DIC, and the marginal likelihood on selecting the correct number of mixture components via a simulation study. We generate 100 simulated data sets with $\pi=0.5$ for different $n$ and $p$ with the true number of components equal to 3. In each stimulated data set, the estimated number of components is determined respectively in terms of the minimum values of AIC, BIC, BIC-ICL, DIC, and the maximum value of the marginal likelihood. Table 4 displays the frequencies of the estimated numbers of components for each method. It demonstrates that the information criteria can be used to determine the number of components. Moreover, we find that even if the assumed number of components is larger than the true number, the Gibbs sampler still tends to select the correct number of components by assigning no observations to the extra components. Evidently, more observations result in higher accuracy in determining the number of components and classifying the observations into these components.

Finally to demonstrate the capability of the proposed approach to handle the problems of large $p$, we consider $p=1000$ and $n=500$. The values of parameters are the same as in the setting above with $M=3$. The value of $g_{m}$ is taken to be the sample size of each group because the ratio of $p$ to $n$ is less than 3. Also, we take the value of $\lambda_{m}=1 / p$ as suggested in the experiment 
Table 3: The computing time in seconds for different scenarios.

\begin{tabular}{ccccc}
\hline$p$ & 25 & 50 & 100 & 150 \\
CPU Time (seconds) & 1079 & 2829 & 5618 & 7805 \\
\hline
\end{tabular}

Table 4: The true number of mixture components is 3. Each cell shows the frequency of the estimated number of components over 100 simulated data sets where the number of components is determined by AIC, BIC, BIC-ICL, DIC and marginal likelihood respectively. We allow the number of components to be chosen from $\{3,4,5\}$

\begin{tabular}{|c|c|c|c|c|c|c|}
\hline \multirow[b]{2}{*}{$M$} & \multicolumn{3}{|c|}{$n=150, p=50$} & \multicolumn{3}{|c|}{$n=300, p=100$} \\
\hline & 3 & 4 & 5 & 3 & 4 & 5 \\
\hline $\mathrm{AIC}$ & 94 & 1 & 5 & 95 & 1 & 4 \\
\hline $\mathrm{BIC}$ & 94 & 4 & 2 & 96 & 3 & 1 \\
\hline BIC-ICL & 95 & 3 & 2 & 97 & 3 & 0 \\
\hline DIC & 94 & 1 & 5 & 96 & 2 & 2 \\
\hline Marginal Loglikelihood & 92 & 4 & 4 & 95 & 3 & 2 \\
\hline
\end{tabular}

we conducted above. For illustration, only a simulated data set with the pair correlation between covariates $\pi=0.5$ is used to study the performance. For this data set, the four measures are shown in Table 5. The result shows that the proposed approach can be applied to the large $p$ problem.

\section{Real Example}

In addition to the simulation studies, a real data set is analyzed to demonstrate the ability of the proposed approach to identify important variables. The date set is about baseball salary, where the variables exhibit high correlations. The data set was analyzed in Khalili and Chen (2007).

The baseball salary data contains 337 major league baseball players' salaries (in thousand) in the year 1992 with 16 performance measures from the year 1991. The players, excluding pitchers, played at least one game in both the 1991 and 1992 seasons. The main interest in this analysis is to detect which performance measures play important roles in determining the salaries.

The 16 performance measures are: batting average $\left(x_{1}\right)$, on-base percentage $\left(x_{2}\right)$, runs $\left(x_{3}\right)$, hits $\left(x_{4}\right)$, doubles $\left(x_{5}\right)$, triples $\left(x_{6}\right)$, home runs $\left(x_{7}\right)$, runs batted in $\left(x_{8}\right)$, walks $\left(x_{9}\right)$, strikeouts $\left(x_{10}\right)$, stolen bases $\left(x_{11}\right)$, and errors $\left(x_{12}\right)$; and indicators of free agency eligibility $\left(x_{13}\right)$, free agent in $1991 / 2\left(x_{14}\right)$, arbitration eligibility $\left(x_{15}\right)$, and arbitration in $1991 / 2\left(x_{16}\right)$. We standardize the explanatory variables, $x_{i}$, for $i=1, \ldots, 12$, in our analysis. The last four dummy variables indicate the degree of freedom for the player to move to another team. Watnik (1998) suggested that there could be potentially important interactions between the quantitative variables $x_{1}, x_{3}, x_{7}$, and $x_{8}$ and the last four variables about the freedom of a player. Among the predicator variables, $x_{1}$ and $x_{7}$ measure the performance of a player, while $x_{3}$ are $x_{8}$ measure the contributions of a player to the team. The total number of variables that may affect a player's salary is 32, including the original variables and their interactions.

Since the salaries are highly right-skewed, a log transform is used to make $\log (\operatorname{salary})$ as the response variable. Khalili and Chen (2007) suggested that a mixture of two normal linear models 
Table 5: The measures of TCR, TPR, and FPR for large $p$ and small $n$ case.

\begin{tabular}{cccc}
\hline TCR & TPR & FPR & TCO \\
\hline 0.99 & 1 & 0.008 & 0.93 \\
\hline
\end{tabular}

should be a proper model assumption, i.e.

$$
y=\log (\text { salary }) \sim \rho N\left(x^{\prime} \beta_{1}, \sigma_{1}^{2}\right)+(1-\rho) N\left(x^{\prime} \beta_{2}, \sigma_{2}^{2}\right)
$$

where $x$ is a $33 \times 1$ vector consisting of all 32 potential covariates plus an intercept. MixLASSO and MixSCAD were used by Khalili and Chen (2007) to identify the important variables. Unlike the homogeneous variance assumption in Khalili and Chen (2007), we assume different variances $\sigma_{1}$ and $\sigma_{2}$.

To analyze this baseball salary data, the setup of the prior parameters are as follows. First the prior inclusion probability, $d_{m j}$, is fixed at 0.5 for all $m$ and $j$. For the inverse gamma prior of $\sigma$, we still set $a_{m_{0}}=b_{m_{0}}=0.001$, which is a nearly non-informative prior. The $\alpha_{i}$ 's in the Dirichlet prior are fixed at 2. For the parameters $\lambda_{m}$ and $g_{m}$ in the $g$-prior, from our pilot study, we find that the singularity problem among the variables does exist, thus we set the ridge parameter $\lambda_{m}=1 / p=1 / 33$ for all $m$. For the $g_{m}$ in the $g$-prior, since the number of observations, $n$, is larger than that of the covariates, $p$, we choose $g_{m}=n=337$. For sensitivity analysis, we also try different values of $g_{m}$, e.g., $10,100,500,1000$, but no significant difference is detected. 1,000,000 sweeps of the Gibbs sampling is implemented to generate the posterior samples of $z_{i}$ and $r_{m j}$.

Based on the estimates of the posterior inclusion probabilities in Figure $5(\mathrm{a})$, we conclude that RBIs plays an important role in achieving a high salary in both sub-populations, according to the median probability criterion. RBI is a measure of the contribution of a player to a team. The more RBI a player has, the more likely the team would win a game. It partly explains that a player with a high RBI commanded a high salary. Figure 2(a) evidently shows that the more RBIs a player contributes to, the higher salary he has in the first sub-population. We also find that Runs is another factor that affects a player's salary in the second population. Although RBIs and Runs are highly correlated (0.88), they both play a role in affecting a player's salary. Moreover, the most significant difference between the two sub-populations are the factors of eligibility for free agency and arbitration. Being a free agent and having the eligibility for arbitration lead to a higher salary in the second sub-population, see Figure 2(b). On the contrary, neither variable is helpful to get a higher pay for the players in the first sub-population. The visualization of the two variables affecting a player's salary are shown in Figure 3 for each sub-population. Compared to the selection results in Khalili and Chen (2007), the number of variables selected by the proposed approach is less than theirs, but the proposed model has more potential to explain the patterns across sub-populations as will be described next.

Based on our results, we classify the observations into different sub-populations, and then fit the linear model for each sub-population using the corresponding variables selected by our method to obtain the adjusted $R^{2}$ value. Compare with the model fitting results by MixLASSO and MixSCAD in Khalili and Chen (2007), the performance of our method is better than those obtained from MixSCAD and MixLASSO in terms of the values of $R^{2}$. It suggests that our approach classifies the observations well so that the observations classified into the same sub-population behave in a similar way. See Table 6. We find that the estimated proportions for the sub-populations are not significantly different in different estimation procedures, such as Bayes, MixSCAD, and MixLASSO. 
Table 6: Posterior estimates of mixing proportions and variances. "Bayes" denotes our results. MIXLASSO and MIXSCAD are the results from (Khalili and Chen 2007).

\begin{tabular}{|c|c|c|c|c|c|c|}
\hline \multirow[b]{2}{*}{ covariate } & \multicolumn{2}{|c|}{ Bayes } & \multicolumn{2}{|c|}{ MIXSCAD } & \multicolumn{2}{|c|}{ MIXLASSO } \\
\hline & group 1 & group 2 & group 1 & group 2 & group 1 & group 2 \\
\hline$\rho$ & 0.28 & 0.72 & 0.24 & 0.76 & 0.28 & 0.72 \\
\hline$\sigma$ & 0.58 & 0.30 & \multicolumn{2}{|c|}{0.32} & \multicolumn{2}{|c|}{0.25} \\
\hline$R^{2}$ & 0.99 & 0.99 & 0.90 & 0.94 & 0.95 & 0.96 \\
\hline
\end{tabular}

But the variances in different sub-populations are clearly different as shown in Figure 4 . Therefore, it is more reasonable to assume different variances for different sub-populations.

Here we set the number of sub-populations at 2 according to the suggestion of Khalili and Chen (2007). To check this assumption, we first use the information criteria studied in Section 2.6 to determine the number of sub-populations. Table 7 shows the estimated information criteria with respect to the number of mixture components. These criteria suggest that it is appropriate to fit the 2-component model to the data.

For a more thorough investigation, we rerun our analysis by assuming 3 sub-populations or components. We classify the observations into the 3 groups, and we order the groups according to the numbers of observations in the groups in the descending order. The posterior inclusion probabilities of the variables under the 3-component model and the 2-component model are shown in Figure 5(b). Based on the median probability criterion, the variables selected are the same for the first two groups in both the 2-component model and the 3-component model. It is worth noting that group 3 of the 3 -component model turns out to be empty. This confirms that the 2 -component model is the correct one.

Table 7: The estimated values of information criteria for different models. NA: not applicable.

\begin{tabular}{ccccc}
\hline \multirow{2}{*}{ Information } & & \multicolumn{3}{c}{ Number of Comonents } \\
& & 2 & 3 & 4 \\
\cline { 5 - 6 } \cline { 5 - 5 } AIC & & 502 & 579 & 604 \\
BIC & & 540 & 621 & 658 \\
DIC & & 1 & 43 & 54 \\
Marginal LogLikelihood & -241 & -279 & -288 \\
BIC-ICL & 760 & 858 & 901 \\
\hline
\end{tabular}

\section{Discussion}

In this section, we discuss several issues about the proposed Bayesian variable selection approach. First, we shall describe how to estimate the regression coefficients based on the posterior samples. Then we shall study the issue of the number of iterations in MCMC and the performance of the proposed algorithm with and without the burn-in step. Finally we shall compare the performances 
Table 8: The estimates of non-zero coefficients and parameters

\begin{tabular}{|c|c|c|c|c|c|c|}
\hline \multirow[b]{2}{*}{ Parameters } & \multicolumn{2}{|c|}{ Component 1} & \multicolumn{2}{|c|}{ Component 2} & \multicolumn{2}{|c|}{ Component 3} \\
\hline & True & Estimate & True & Estimate & True & Estimate \\
\hline$\beta_{1}$ & 3 & 3.06 & 0 & 0 & 0 & 0 \\
\hline$\beta_{2}$ & 3 & 2.84 & 0 & 0 & 0 & 0 \\
\hline$\beta_{3}$ & 0 & 0 & -2 & -2.04 & 0 & 0 \\
\hline$\beta_{4}$ & 0 & 0 & -2 & 2.00 & 0 & 0 \\
\hline$\beta_{5}$ & 0 & 0 & 0 & 0 & -3 & -2.92 \\
\hline$\beta_{6}$ & 0 & 0 & 0 & 0 & 2 & 1.96 \\
\hline$\rho$ & $1 / 3$ & 0.32 & $1 / 3$ & 0.34 & $1 / 3$ & 0.34 \\
\hline$\sigma$ & 0.50 & 0.45 & 0.50 & 0.51 & 0.50 & 0.61 \\
\hline
\end{tabular}

of two different model selection criteria, the median posterior probability and the highest posterior probability, on variable selection via a stimulation study.

\subsection{Posterior inference of regression coefficients}

Since we focus on variable selection, we would identify the active variables first and then reestimate the corresponding coefficients by setting to zeros the coefficients of those inactive variables. In the Step 4 of the proposed Gibbs sampler, we still generate samples of the coefficients, $\beta_{m}$. Thus we can estimate these active coefficients based on the posterior samples.

Given the posterior samples of the coefficients, the posterior mean $\mathrm{E}(\beta \mid y)$ is a natural estimate of the coefficients. In fact, the posterior mean can be approximated in a straightforward manner from simulated samples. We use Rao-Blackwellization (Gelfand and Smith, 1990) to estimate the regression coefficient $\beta_{m j}$

$$
\mathrm{E}\left(\beta_{m j} \mid y\right)=\sum_{r_{m j}} E\left[\beta_{m j} \mid r_{m j}, y\right] p\left(r_{m j} \mid y\right) \approx \frac{1}{K_{j}} \sum_{k=1}^{K} \beta_{m j}^{[k]},
$$

where $K_{j}=\sum_{k=1}^{K} r_{m j}^{[k]}, r_{m j}^{[k]}$ and $\beta_{m j}^{[k]}$ are the MCMC samples in the $k$ th iteration.

We revisit Simulation 2 with $p=50$ and $n=150$, and the pair correlation $\pi=0.5$. We investigate the accuracy of the posterior estimates for the parameters by comparing the estimates to the true values. Based on the same set-up as in Simulation 2, that is $\lambda=1 / p$ and $g_{m}=n_{m}$, $1,000,000$ samples are generated by the Gibbs sampler. The estimates of $\beta$ 's are given in Table 8 . The estimates of the non-zero coefficients are close to the true parameter values. In addition to the coefficients, the posterior means of $\sigma$ 's and $\rho$ 's are also shown in Table 8

\subsection{The convergence property and burn-in step}

We assess the convergence of the proposed Gibbs sampler based on the Monte Carlo standard error (MCSE) in Flegal et al. (2008). Specifically, we check the convergence of the proposed MCMC algorithm by tracing the corresponding MCSE of $\sigma$ 's. The MCMC chain stops whenever the largest MCSE of the estimates of $\sigma$ 's is less than 0.005, following the suggestion of Flegal et al. (2008). We revisit Simulation 2 with $p=50, n=150$ and $\pi=0.5$ to study the issue of convergence and burn-in step. Using the same tuning parameters as before, that is, $g_{m}=n_{m}$ and $\lambda=1 / p$, 
Table 9: The cumulative estimates of $\sigma$ 's and the corresponding MCSEs for every 10,000 iterations.

\begin{tabular}{|c|c|c|c|c|c|c|}
\hline \multirow[b]{2}{*}{ Iteration } & \multicolumn{2}{|c|}{ Component 1} & \multicolumn{2}{|c|}{ Component 2} & \multicolumn{2}{|c|}{ Component 3} \\
\hline & $\hat{\sigma}_{1}$ & $\operatorname{MCSE}\left(\hat{\sigma}_{1}\right)$ & $\hat{\sigma}_{2}$ & $\operatorname{MCSE}\left(\hat{\sigma}_{2}\right)$ & $\hat{\sigma}_{3}$ & $\operatorname{MCSE}\left(\hat{\sigma}_{3}\right)$ \\
\hline $1.0 \mathrm{E}+04$ & 0.46 & 0.00942 & 0.54 & 0.02480 & 0.65 & 0.03750 \\
\hline $2.0 \mathrm{E}+04$ & 0.45 & 0.00502 & 0.52 & 0.01400 & 0.62 & 0.02210 \\
\hline $3.0 \mathrm{E}+04$ & 0.45 & 0.00345 & 0.52 & 0.01040 & 0.61 & 0.01570 \\
\hline $4.0 \mathrm{E}+04$ & 0.45 & 0.00266 & 0.52 & 0.00850 & 0.61 & 0.01270 \\
\hline $5.0 \mathrm{E}+04$ & 0.45 & 0.00214 & 0.51 & 0.00690 & 0.61 & 0.01100 \\
\hline $6.0 \mathrm{E}+04$ & 0.45 & 0.00179 & 0.51 & 0.00570 & 0.60 & 0.00980 \\
\hline $7.0 \mathrm{E}+04$ & 0.45 & 0.00154 & 0.51 & 0.00490 & 0.60 & 0.00840 \\
\hline $8.0 \mathrm{E}+04$ & 0.45 & 0.00135 & 0.51 & 0.00430 & 0.60 & 0.00740 \\
\hline $9.0 \mathrm{E}+04$ & 0.45 & 0.00121 & 0.51 & 0.00380 & 0.60 & 0.00650 \\
\hline $1.0 \mathrm{E}+05$ & 0.45 & 0.00109 & 0.51 & 0.00340 & 0.60 & 0.00590 \\
\hline $1.1 \mathrm{E}+05$ & 0.45 & 0.00099 & 0.51 & 0.00310 & 0.60 & 0.00540 \\
\hline $1.2 \mathrm{E}+05$ & 0.45 & 0.00091 & 0.51 & 0.00290 & 0.60 & 0.00490 \\
\hline$\vdots$ & $\vdots$ & $\vdots$ & $\vdots$ & $\vdots$ & 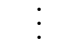 & : \\
\hline $2.0 \mathrm{E}+05$ & 0.45 & $<0.00001$ & 0.51 & $<0.00001$ & 0.60 & $<0.00001$ \\
\hline$\vdots$ & $\vdots$ & $\vdots$ & $\vdots$ & $\vdots$ & $\vdots$ & $\vdots$ \\
\hline $1.0 \mathrm{E}+06$ & 0.45 & $<0.00001$ & 0.51 & $<0.00001$ & 0.60 & $<0.00001$ \\
\hline
\end{tabular}

$1,000,000$ samples are generated for the simulated data. Actually the stopping rule suggests that it is not necessary to have such a long chain. For instance, the chain would be stopped after 120,000 iterations when all the MCSEs of the estimates of $\sigma$ 's are less than 0.005. Putting together the findings from Table 9 and the visual assessment in Figure 6, we can conclude that the MCMC outputs indicate convergence. Once the largest MCSE of $\sigma$ 's is less than 0.005, the chain will stop and the samples are used to estimate the parameters of interest.

We further investigate the the effect of the burn-in step on the parameter estimation. Flegal et al. (2008) suggested that we might not need to have the burn-in step if the starting values are reasonable. In fact, we never know if the starting value is good or not. Thus we first run multiple chains by randomly assigning the starting values of $\gamma$ 's. We find that different chains produce almost the same Bayesian inference results. Thus we believe that the null model is a reasonable initialization. To see the effect of the burn-in step, we compare the estimates of $\sigma^{2}$ 's with and without the burn-in step. Based on previous study on the stopping rule, the chain is stopped at 120,000 iterations. We then burn in 10,000 and 20,000 samples for estimation. Table 10 shows the estimates of $\sigma$ 's by a chain with and without the burn-in step. It can be seen that the estimates of $\sigma$ 's in three different components are identical with or without burn-in. In fact, all results from different examples in the paper are essentially the same either with or without the burn-in step. Thus probably the burn-in step can be waived here, and we believe, at least in our examples, the burn-in step is not necessarily required.

\subsection{Selection criteria}

In this paper, the median probability criterion in 20 is used to identify the proper variables in each subgroup. The highest posterior probability criterion is also a commonly used criterion. 
Table 10: The estimates of $\sigma$ 's with and without burning in the samples.

\begin{tabular}{cccc}
\hline Burn In & $\hat{\sigma}_{1}$ & $\hat{\sigma}_{2}$ & $\hat{\sigma}_{3}$ \\
\hline 0 & 0.45 & 0.51 & 0.60 \\
10,000 & 0.45 & 0.51 & 0.60 \\
20,000 & 0.45 & 0.51 & 0.60 \\
\hline
\end{tabular}

Table 11: The measures of TCR, TPR, and FPR from two different selection criterion.

\begin{tabular}{ccc} 
& Median Probability & Highest Posterior Probability \\
\hline TCR & 0.99 & 0.98 \\
TPR & 1 & 1 \\
FPR & 0.010 & 0.018 \\
\hline
\end{tabular}

Based on the highest posterior probability criterion, the set of variables is selected by maximizing the posterior probability among all $2^{p}$ possible models. Barbieri and Berger (2004) have shown that under certain conditions, both criteria can identify the same model for the linear regression.

We compare these two selection criteria using a simulation study. The data is generated by following Simulation 2 with $M=3, n=150, p=50$ and $\pi=0.5$. Using the same tuning parameters in Simulation 2, the proposed Gibbs sampler is iterated 1,000,000 sweeps to generate the samples. After 100 replications, the selection results obtained by these two different criteria are shown in Table 11. Judging from the three performance measures, the two different selection approaches perform equally well and their results are similar to each other.

In addition to these three performance measures, we also examine the ranking of the regression model in each component identified by the median probability approach in terms of its posterior probability. Over 100 simulated data sets, the average rankings for the three components are 1.1 (0.25), $1.1(0.23), 1.2(0.40)$, respectively, where the standard deviations are in the parentheses. This result shows that the model selected by the median probability criterion is in the top ranking in terms of the posterior probabilities. Thus we believe that both criteria would select very similar models. Based on the computational efficiency, we would suggest using the median probability criterion.

\section{Conclusions}

In this article, we develop a Bayesian variable selection method for the finite mixture model of linear regressions. Two different sets of indicators for the observations and variables are augmented into the model, and a Gibbs sampler is proposed to generate the posterior samples so that they can be used to infer the active variables within different components or sub-populations. For the $g$-prior assumption, we demonstrate how to incorporate the ridge regression that allows for the case where the model matrix is not of full rank. A further extension for the $g$-prior is the use of the mixture of $g$-priors (Liang, et al. , 2012). Our method can be applied to the situation where the number of predictor variables is larger than the number of observations, a problem that has become increasingly common in practice.

We have investigated the problem of determining the number of sub-populations or mixture components, $M$, using various information criteria. These criteria perform well for identifying the 
number of components. With a prior on the number of components, a fully Bayesian approach powered by the reversible jump MCMC algorithm (Richardson and Green, 1997, Tadesse et al. 2005) can be used to infer the number of components while selecting the variables for each component. But such an approach may dramatically increase the computational cost since the MCMC algorithm requires extra steps such as birth and death, splitting and merging, etc. All of them need Metropolis-Hastings algorithm within the Gibbs sampling procedure. A recent implementation of the reversible jump MCMC for Bayesian variable selection is Liu et. al (2014) where they studied the performance of weighted $g$-prior on variable selection. We shall extend their approach to our problem in our future work.

Yau and Holmes (2011) and Chung and Dunson (2009) studied the hierarchical Bayesian nonparametric mixture model, where the number of mixture components, $M$, is inferred based on the posterior distribution. However, they did not consider the problems of $p>n$ and high collinearity. We shall extend their methods to address these problems in our future work.

In addition to variable selection issue, we can extend the proposed approach for other interesting problems. Firstly, the proposed variable selection approach is not limited to the finite mixture of regression models. It can be easily extended to the latent class models (Ghosh et al. , 2011) with

$p$ larger than $n$. Secondly, similar to Tran et al. (2012), expert knowledge can be incorporated into the proposed model through the prior distribution of the proportion of observations in each sub-population. This may increase the accuracy of classification.

\section{Acknowledgments}

The research of Chen and Lee is supported in part by the National Science Council under grant NSC 99-2118-M-006-006-MY2 and MOST 103-2118-M-006-002-MY2 (Chen) and NSC 101-2118M-006-007- (Lee), and the Mathematics Division of the National Center for Theoretical Sciences (South) in Taiwan.

The authors would like thank the referees, the associate editor and the editor for their insightful and constructive comments that have substantially improved this article.

\section{References}

Baragatti, M., Pommeret, D. (2012). A Study of Variable Selection Using g-prior Distribution with Ridge Parameter. Computational Statistics and Data Analysis, 56:1920-1934.

Barbieri, M., Berger, J. O. (2004). Optimal Predictive Model Selection, Annals of Statistics, 32:870897.

Biernacki, C., Celeux, G., Govaert, C. (2000). Assessing a Mixture Model for Clustering with the Integrated Classification Likelihood, IEEE Transactions on Pattern Analysis and Machine Intelligence, 22: 719-725.

Celeux G. (1998). Bayesian Inference for Mixture: The Label Switching Problem, COMPSTAT, $227-232$

Celeux G., Forbes F., Robert C.P., Titterington D.M. (2006). Deviance Information Criteria for Missing Data Models, Bayesian Analysis, 4:651-674. 
Chen B., (2012). Bayesian model selection in finite mixture regression, Dissertations \& Theses Gradworks, http://gradworks.umi.com/35/48/3548634.html

Y. Chung Y., Dunson D.B. (2009). Nonparmetric Bayes Conditional Distribution Modeling With Variable Selection, Journal of the American Statistical Association, 104:1646-1660.

Cohen, E. (1980). Inharmonic Tone Perception, Ph.D Dissertation, Stanford University.

Fan J., Li, R. (2001). Variable Selection via Non-Concave Penalized Likelihood and its Oracle Properties, Journal of the American Statistical Association, 96:1348-1360.

Flegal J. M., Haran M., Jones, G. L. (2008). Markov Chain Monte Carlo: Can We Trust the Third Significant Figure?, Statistical Science, 23:250-260.

Sylvia Frühwirth-Schnatter (2006). Finite Mixture and Markov Switching Models, Springer, 2006.

Gelfand A.E., Smith A.F.M. (1990). Sampling-based approaches to calculating marginal densities, Journal of the American Statistical Association, 85:398-409.

George, E. I., McCulloch, R. E. (1993). Variable Selection via Gibbs Sampling, Journal of the American Statistical Association, 88:881-889.

Gupta M., Ibrahim, J. (2007). Variable Selection in Regression Mixture Modeling for the Discovery of Gene Regularory Nectworks, Journal of the American Statistical Association, 102:867-880.

Ghosh J., Herring A.H., Siega-Riz A.M. (2011). Bayesian Variable Selection for Latent Class Models, Biometrics, 67:917-925.

Jasra A, Holmes C.C., Stephens D.A. (2005). Markov Chain Monte Carlo Methods and the Label Switching Problem in Bayesian Mixture Modeling, Statistical Science, 20:50-67.

Khalili, A., Chen, J. (2007). Variable Selection in Finite Mixture of Regression Models, Journal of the American Statistical Association, 102:1025-1038.

Liang F., Paulo R. and Molina G. and Clyde M.A. and Berger J.O. (2012). Mixtures of $g$ Priors for Bayesian Variable Selection, Journal of the American Statistical Association, 103:410-423

Liu W., Zhang B., Zhang Z, Tao J., Branscum A.J.(2014+). Model selection in finite mixture of regression models: a Bayesian approach with innovative weighted g priors and reversible jump Markov chain Monte Carlo implementation, Journal of Statistical Computation and Simulation, $2014+$.

McLachlan, G. J., Peel D. (2000). Finite Mixture Models, Wiley.

Park, T., Casella, G. (2008). The Bayesian Lasso, Journal of the American Statistical Association, 103:681-686.

Richardson S. and Green P.J. (1997). On Bayesian analysis of mixtures with an unknown number of components, Journal of the Royal Statistical Society: Series B, 59:731-792.

Städler, N., Bühlmann, P., van de Geer, S. (2010). $\ell_{1}$-Penalization for Mixture Regression Models, Test, 19: 209-256. 
Tadesse M.G. Sha N., Vannucci M. (2005). Bayesian Variable Selection in Clustering HighDimensional Data, Journal of the American Statistical Association, 100:602-617.

Tibshirani, R. (1996). Regression Shrinkage and Selection via the LASSO, Journal of the Royal Statistical Society: Series B, 58:267-288.

Tibshirani, R. (2011). Regression Shrinkage and Selection via the Lasso: a Retrospective, Journal of the Royal Statistical Society: Series B, 73:273-282.

Tran M., Nott D. J., Kohn R. (2012). Simultaneous variable selection and component selection for regression density estimation with mixtures of heteroscedastic experts, Electronic Journal of Statistics, 6:1170-1199

Viele K., Tong, B. (2002). Modeling with Mixtures of Linear Regressions, Statistics and Computing, 12:315-330.

Watnik, M. R. (1998). Pay for Play: Are Baseball Salaries Based on Performance?, Journal of Statistics Education, 6, n.2.

Yau C., Holmes, C. (2011). Hierarchical Bayesian nonparametric mixture models for clustering with variable relevance determination, Bayesian Analysis, 6:329-352.

Zellner, A. (1996). On Assessing Prior Distributions and Bayesian Regression Analysis With $g$ prior Distributions. In Bayesian Inference and Decision Techniques: Essays in Honor of Brunode Finetti North-Holland/Elsevier, pp 233-243.

Zhang, C.-H. (2010). Nearly Unbiased Variable Selection under Minimax Concave Penalty, Annals of Statistics, 38:894-942. 


\section{True Positive Rate}

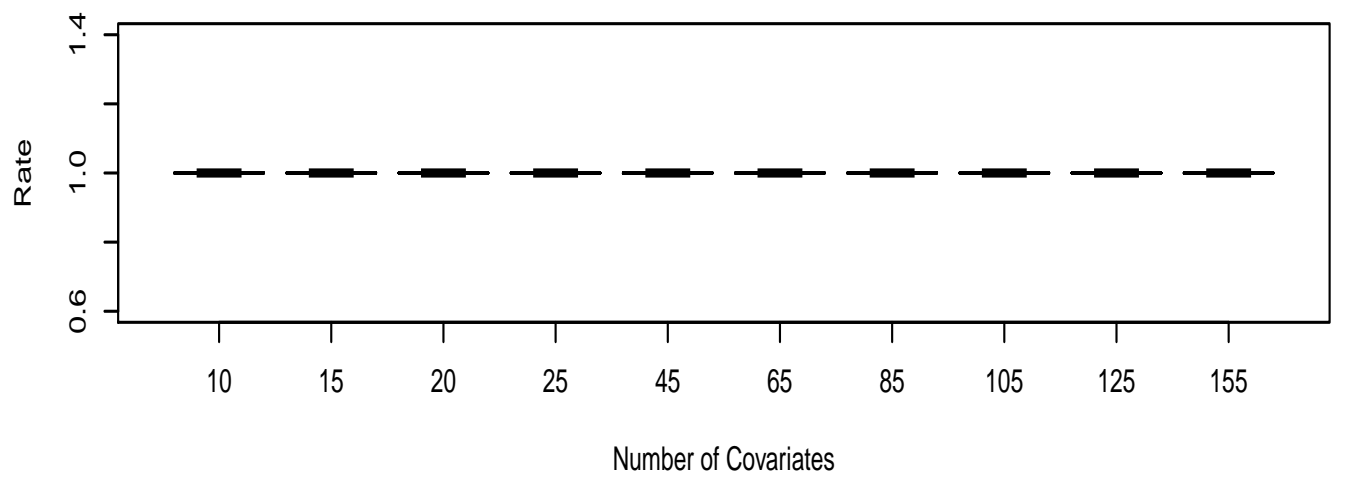

\section{FALSE Positive Rate}

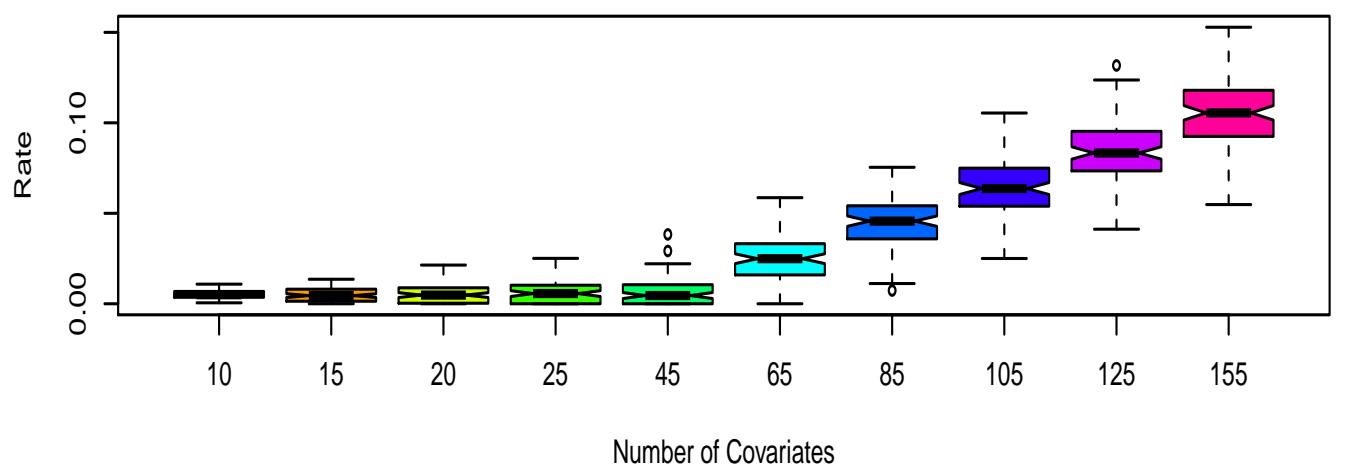

Rate for True Classification of Observations

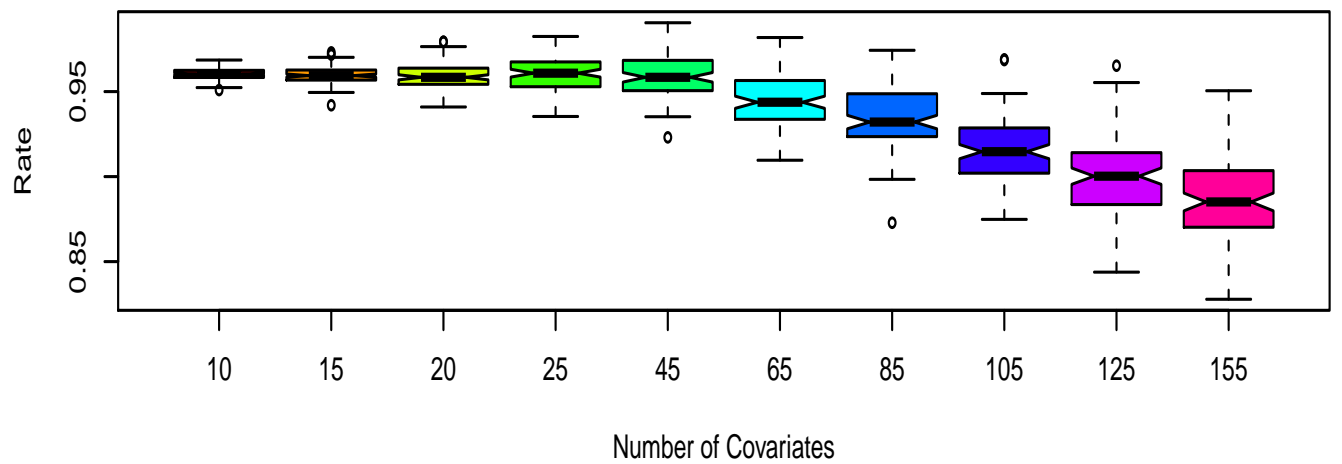

Figure 1: Box plots for the true positive rate (TPR), false 21 positive rate (FPR), and the rate of true classification of observations (TCO), for different numbers of covariates. 


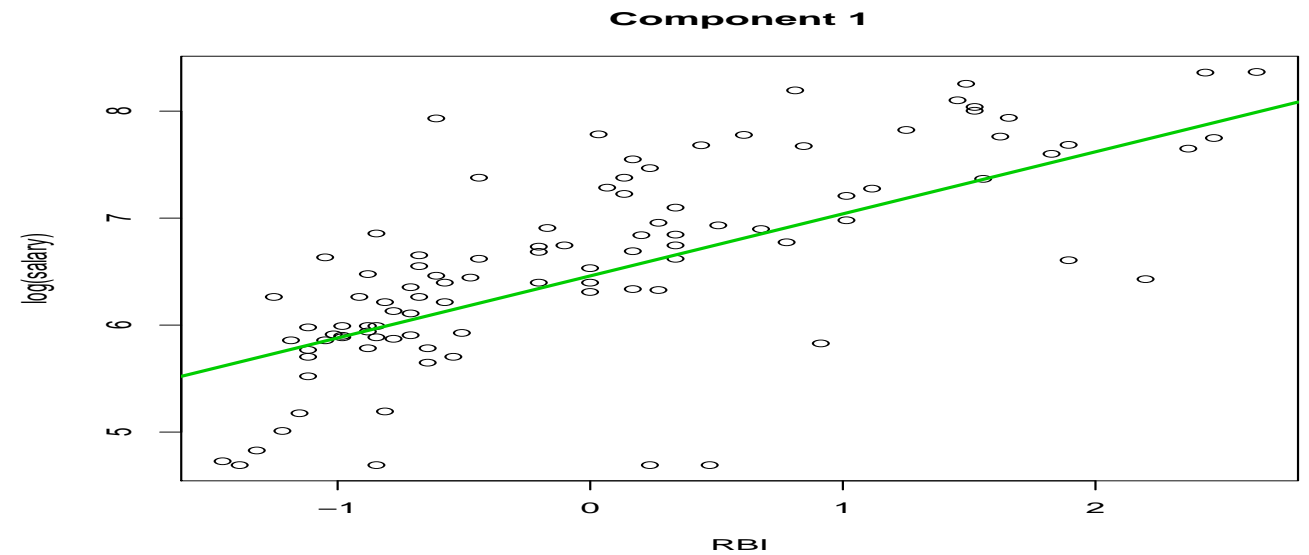

(a) The green line is drawn using the $\beta$ value obtained by our approach.

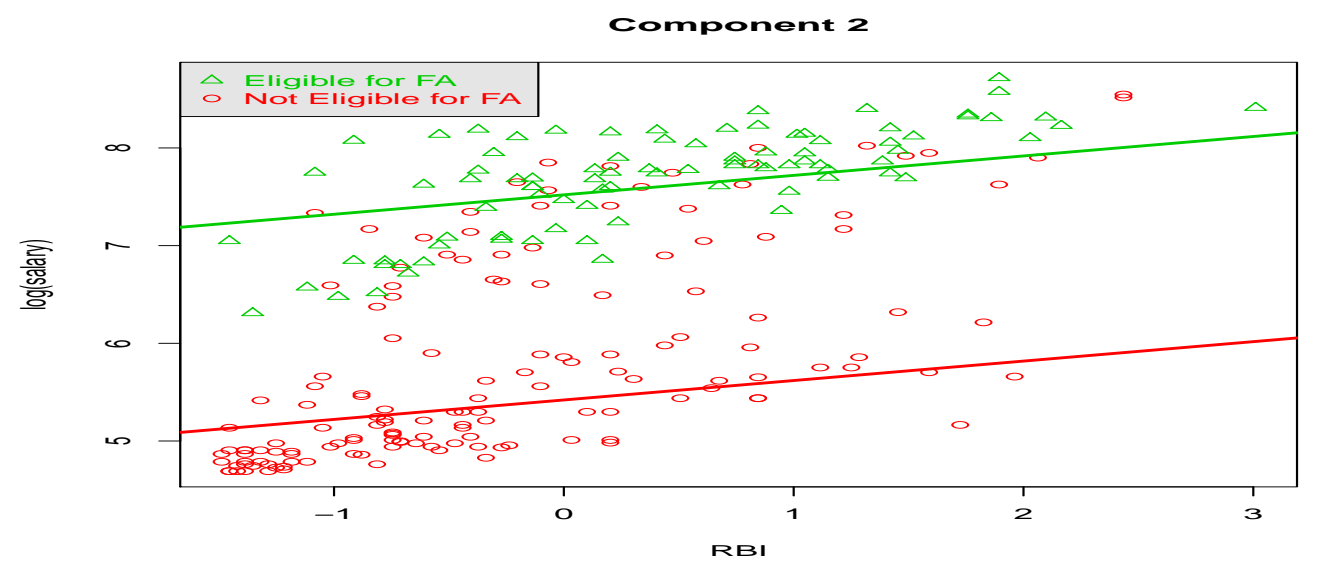

(b) The red/green lines are drawn using the $\beta$ values obtained by our approach. The green points illustrate the players with eligibility for free agency, whereas the red points are the players without such eligibility.

Figure 2: Scatter plot of logarithm of baseball salary versus RBI. 
Component 1
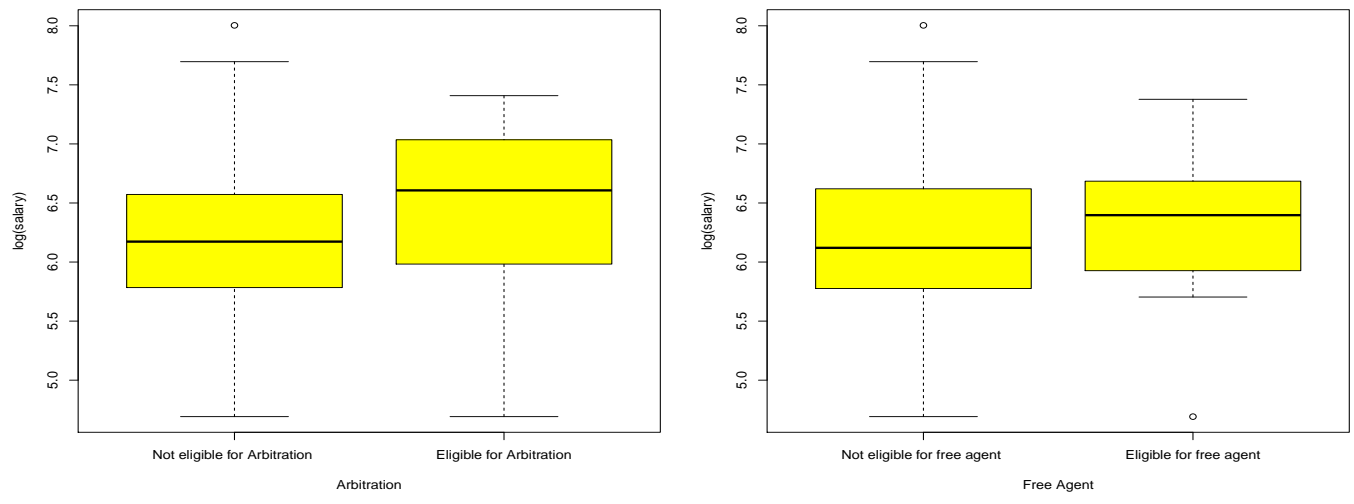

Component 2
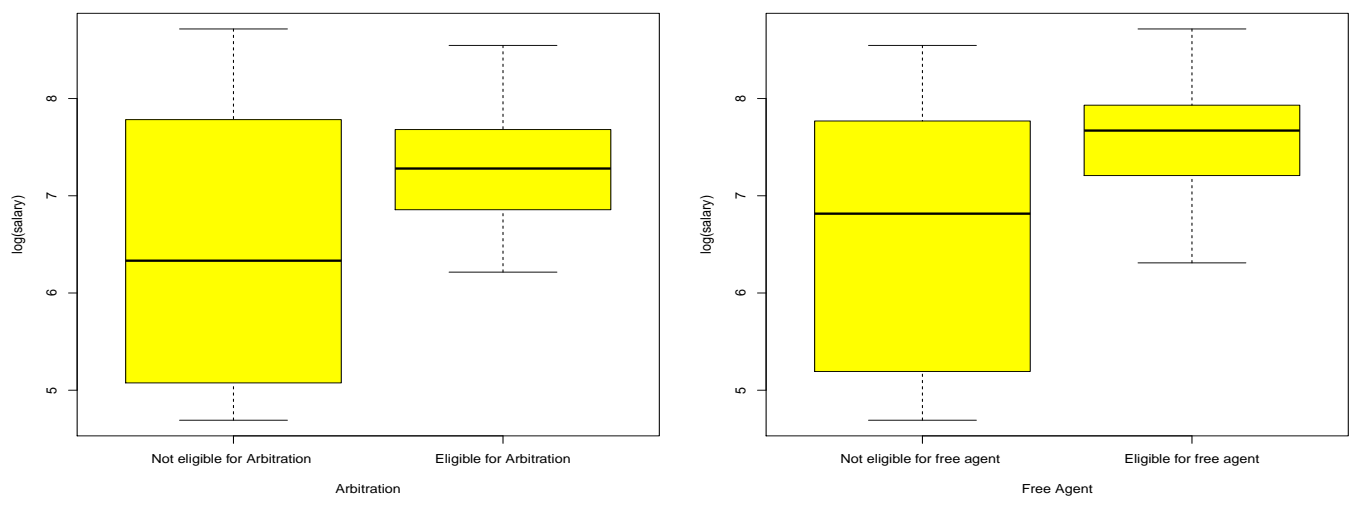

Figure 3: Box plots of salaries based on factors of eligibility for free agency and arbitration in different subpopulations. 


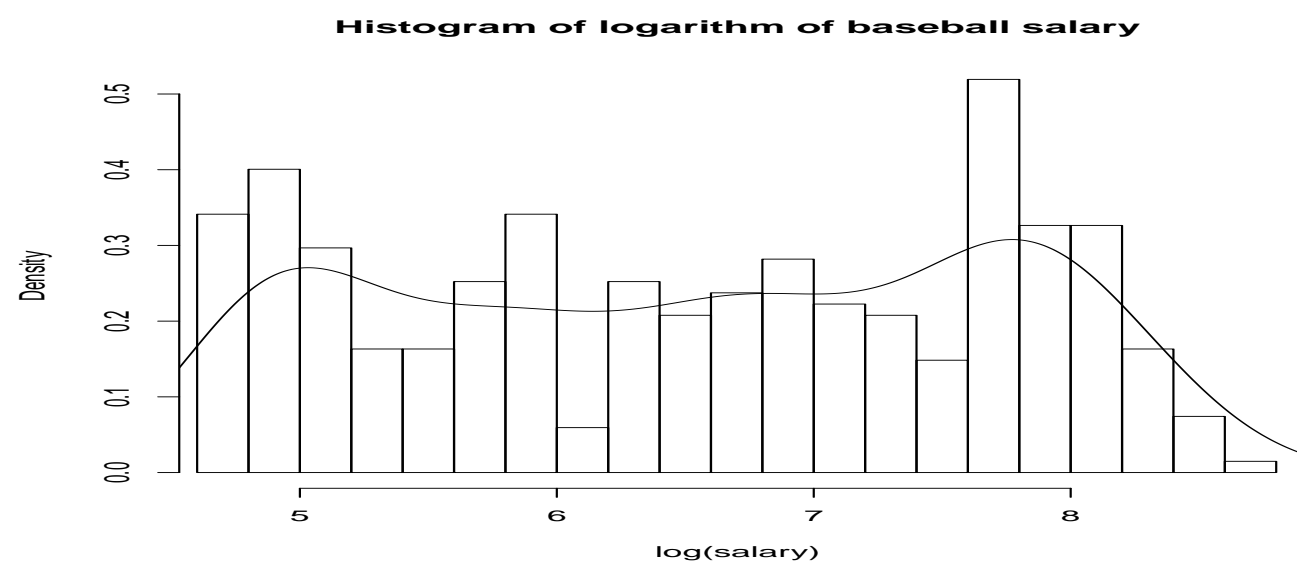

(a) The distribution of raw data of baseball salaries.

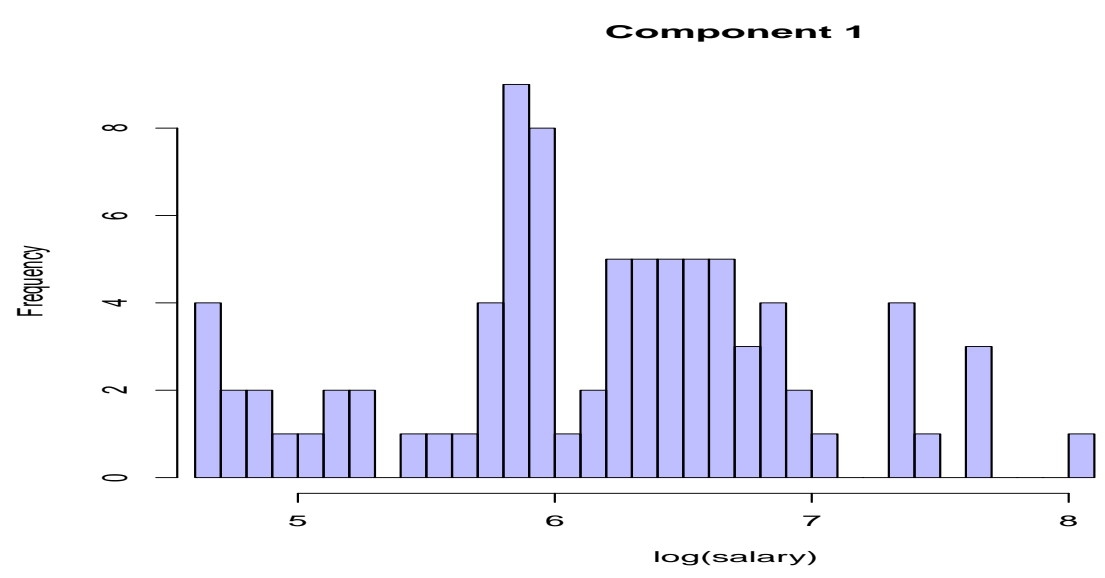

(b) The distribution of observations in the first component.

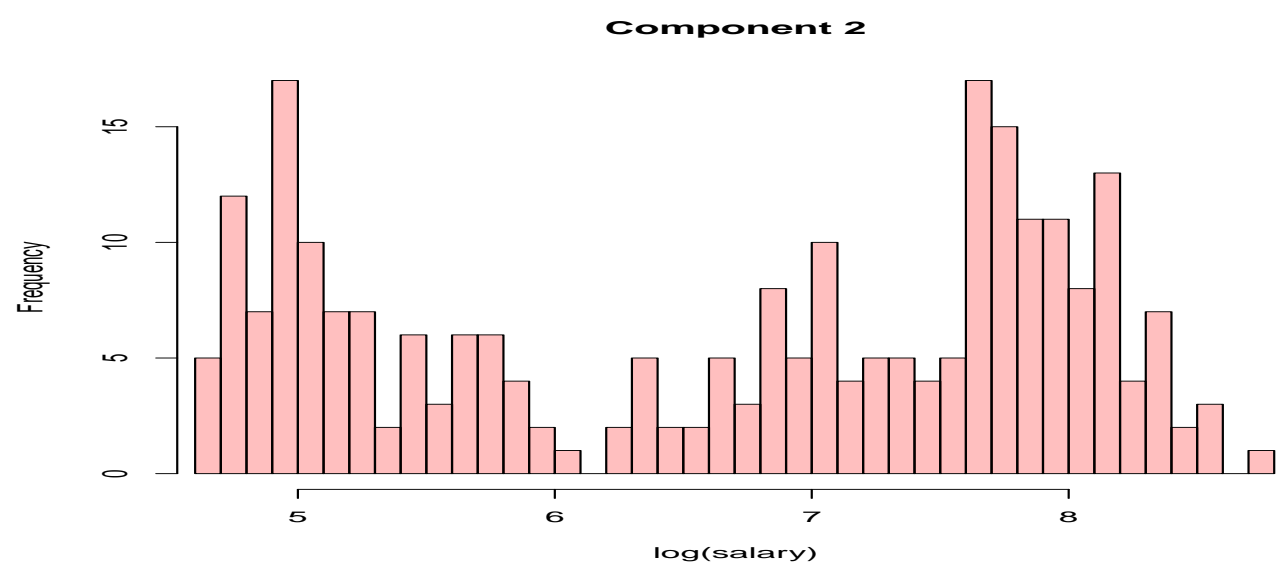

(c) The distribution of observations in the second component.

24

Figure 4: Baseball salary study. 


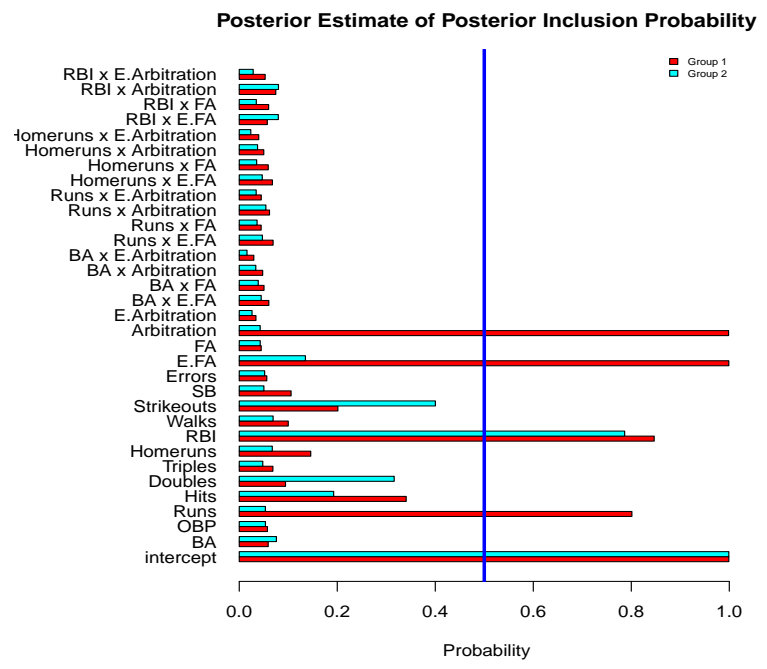

(a) Two components.

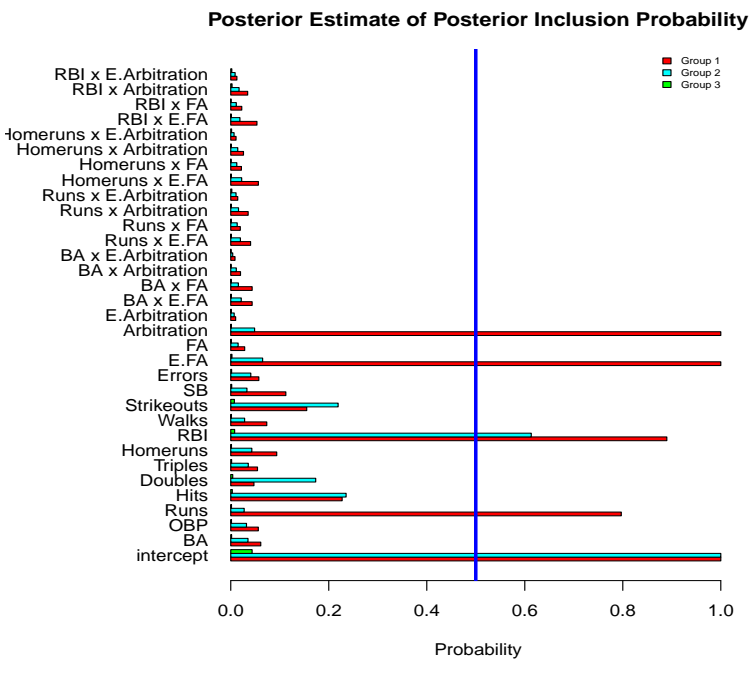

(b) Three components.

Figure 5: The estimated posterior inclusion probability of each variable in each component for 2 different models. The vertical blue line represents the probability of 0.5 . 


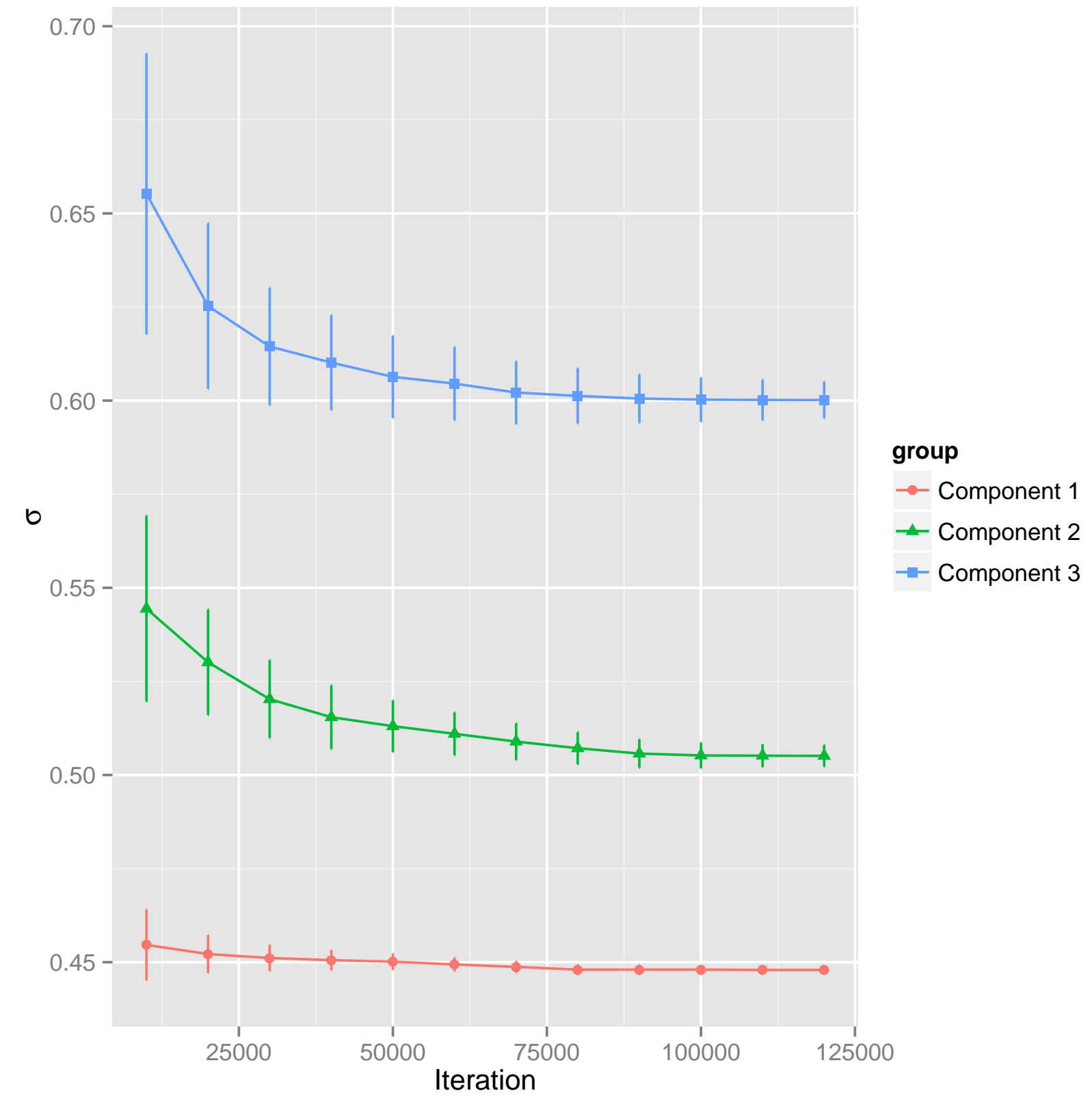

Figure 6: The cumulative estimates of $\sigma$ 's for every 10,000 iterations. The error bars are the estimates of $\sigma$ 's plus and minus twice the MCSEs of the corresponding estimates. 